\title{
UMA REVISÃO BIBLIOGRÁFICA EXPLORATÓRIA SOBRE AFFORDANCES E NORMAS SOCIAIS
}

\author{
Alysson Bolognesi Prado \\ Universidade Estadual de Campinas-Unicamp-Brasil
}

\begin{abstract}
RESUMO
Com o enriquecimento tecnológico de nossa vida cotidiana, a sociomaterialidade de nossas relações se torna cada vez mais evidente. Neste artigo, realizamos uma revisão bibliográfica em busca de fontes teóricas de dois conceitos que nos capacitam a entender como pessoas interagem com objetos no ambiente ao seu redor e com outras pessoas: affordances e normas sociais. Partindo da hipótese que uma visão convergente destes dois conceitos pode ser relevante para abordar problemas contemporâneos da Interação Humano-Computador, o objetivo deste trabalho é trazer um resumo de suas definições e discutir suas convergências e lacunas. Esperamos ter contribuído com uma fonte concisa e simplificada, em língua portuguesa, de referenciais teóricos relevantes para dois dos conceitos mais importantes para pesquisa em IHC e design de aplicações e dispositivos com viés social. Acrescentamos ao estudo resultados relacionados vindos de outras áreas do conhecimento, como neurociência e sociologia. Concluímos que tanto pessoas como objetos podem ser igualmente fontes de influência na disposição para ação de pessoas, e este conceito geral pode ser melhor explorado.
\end{abstract}

\section{PALAVRAS-CHAVE}

Revisão Bibliográfica, Affordances, Normas Sociais, Artefatos Digitais, Computação Social, Interação Humano-Computador

\section{INTRODUÇÃO}

A interação humano-computador ultrapassou os limites do computador enquanto ferramenta em si, provendo objetos computacionalmente melhorados - computationally enhanced devices (Dourish, 2004) - bem como a agentes com variados graus de autonomia para ambiente de trabalho - co-bots (Brooks, 1991) - e fora dele (Birnbaum et al., 2016). Para entender como pessoas interagem com o ambiente ao seu redor e com outras pessoas, dois conceitos teóricos são comumente utilizados: affordances e normas, respectivamente. Entretanto, as diversas fontes teóricas disponíveis para cada um os abordam de maneiras diferentes, propondo definições que podem não ser totalmente convergentes (Kaptelinin e Nardi, 2012).

Neste artigo, realizamos uma revisão bibliográfica em busca das fontes conceituais mais usadas em pesquisa de Interação Humano-Computador (IHC), bem como de propostas alternativas relevantes. Consideramos esta revisão como exploratória, pois não desejamos que ela seja exaustiva, varrendo todo o conjunto de publicações encontrados, ou que siga algum método formal de revisão bibliográfica. Também não estamos interessados propriamente nas publicações encontradas, mas sim nas referências bibliográficas utilizadas, de modo a localizarmos fontes teóricas mais usadas pelos trabalhos que abordam nossos temas de interesse - affordances e normas. O objetivo deste trabalho é iniciar um arcabouço teórico para que, em trabalhos futuros, seja possível responder à questão: como equipar ontologicamente designers e projetistas em um mundo em que a fronteira da relação entre pessoas e dispositivos se torna cada vez mais tênue?

Na próxima seção, apresentaremos o método adotado para localização, seleção e classificação dos textos analisados. Na Seção 3, serão elencados os artigos mais citados sobre affordances e sobre normas, juntamente com um breve resumo das definições propostas ou adotadas; também nesta seção serão mostrados os textos que se encaixam nas duas buscas. Na seção seguinte, mostramos algumas pesquisas recentes de outras áreas que enriquecem a discussão sobre o tema, localizadas independentemente da revisão bibliográfica. A Seção 5 traz a discussão dos resultados obtidos, suas limitações e possibilidades futuras. O artigo se encerra com os comentários finais da Seção 6. 


\section{MÉTODO}

Esta pesquisa foi realizada nas seguintes etapas: iniciou-se com buscas na biblioteca digital da ACM para as palavras-chave "affordances" e "norms", cujos resultados foram inseridos em um software de apoio para o processo de revisão (conjunto bibliográfico inicial I). Trata-se do software Quid (www.quid.net.br) que organiza conjuntos bibliográficos, permitindo registrar os autores, referências citadas, autores destas referências etc., dados que podem ser posteriormente exportados para análise, cruzamentos, filtros e totalizações em outros softwares de planilha eletrônica, por exemplo.

O Quid buscou-se então, de forma automatizada, as referências bibliográficas dos artigos encontrados nas buscas, gerando o conjunto bibliográfico derivado (D). Esta lista de referências, em ordem decrescente do número de referências, foi inspecionada manualmente, em busca de fontes relevantes para os conceitos de interesse (conjunto selecionado S). As publicações que contribuíam conceitualmente foram selecionadas para leitura detalhada e discussão. A seleção dos textos para análise está esquematizada na Figura 1.

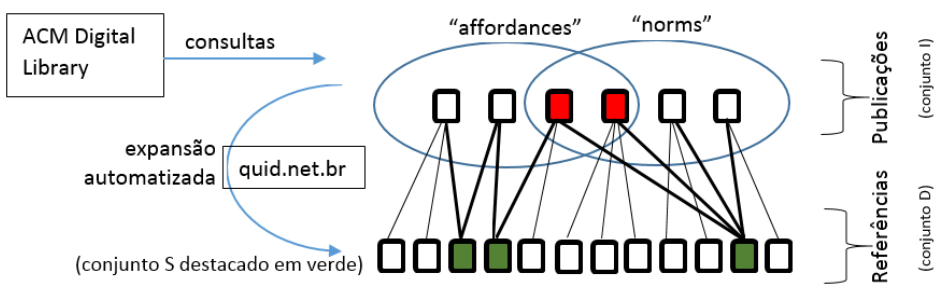

Figura 1. Seleção dos textos para análise. As referências destacadas em verde são tratadas nas Seções 3.1 e 3.2 (conjunto S), enquanto que as publicações marcadas em vermelho são apesentadas na Seção 3.3. Fonte: autor

A busca foi feita em 21/03/2019. Utilizou-se apenas a biblioteca digital da ACM, porque os seus resultados são os que mais facilmente alimentam a ferramenta usada nas etapas seguintes. Ambos os conjuntos de dados podem ser consultados em http://www.quid.net.br/quid/View.jsf?id=1626558 e http://www.quid.net.br/quid/View.jsf?id=1609550. Buscas adicionais em outras bases bibliográficas, pelas mesmas palavras-chave, geraram a Seção 4.

\section{RESULTADOS}

Nesta seção mostramos dados gerais dos levantamentos bibliográficos, bem como a lista detalhada dos artigos escolhidos para discussão. Na Figura 2 a seguir, estão quantificados os artigos encontrados em cada busca.
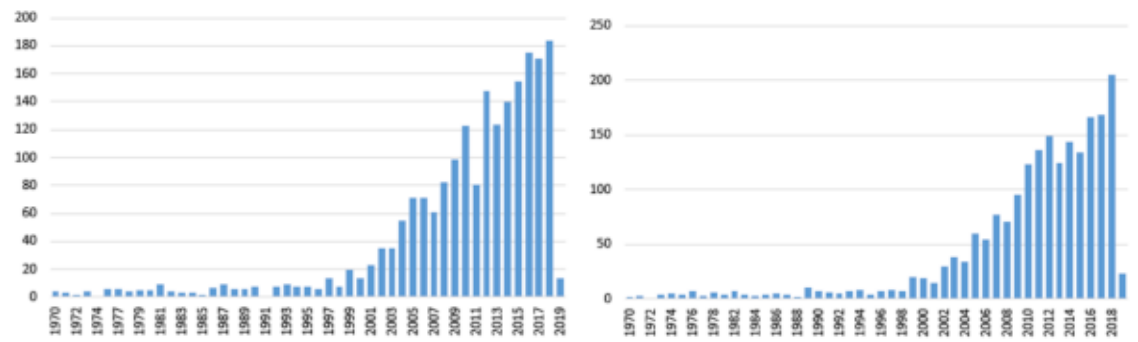

Figura 2. Quantidade por ano de publicação de artigos encontrados na Biblioteca Digital da ACM na busca por "affordance" (à esquerda) e "norm" (à direita). Fonte: autor

\subsection{Affordances}

Foram encontrados 1743 artigos $\left(\mathrm{I}_{\mathrm{A}}\right)$, cuja distribuição por ano de publicação é mostrada na Figura 2 (esquerda). As dez conferências ou periódicos em que foram encontrados com mais frequência são listados na Tabela 1. A partir deste conjunto, foram localizadas 22482 referências distintas $\left(D_{A}\right), 2757$ delas sendo citadas por mais de um artigo do conjunto inicial. Os dez artigos mais citados $\left(\mathrm{S}_{\mathrm{A}}\right)$ são mostrados na Tabela 2 
a seguir. Alguns resultados são mais caracterizáveis como fontes teóricas enquanto outros trazem mais aplicações dos conceitos. Serão apresentados a seguir, na ordem do de maior contribuição teórica até os mais voltados para aplicação. Os textos (A5, A6, A7 e A9) não fornecem definições para affordances e não serão descritos.

Tabela 1. As 10 conferências ou periódicos mais encontrados na busca por affordances. Fonte: autor

\begin{tabular}{llll}
\hline Sigla & Qtd & $\%$ & Nome do periódico/conferência \\
\hline CHI & 151 & $9 \%$ & ACM CHI Conference on Human Factors in Computing Systems \\
\hline CHI EA & 146 & $8 \%$ & CHI Extended Abstracts \\
\hline TEI & 51 & $3 \%$ & International Conference on Tangible, Embedded and Embodied Interaction \\
\hline CSCW & 50 & $3 \%$ & ACM Conference on Computer-Supported Cooperative Work and Social Computing \\
\hline IDC & 43 & $2 \%$ & ACM Interaction Design and Children conference \\
\hline SIG GRAPH & 42 & $2 \%$ & ACM SIGGRAPH annual conference on computer graphics \\
\hline UIST & 36 & $2 \%$ & ACM Symposium on User Interface Software and Technology \\
\hline WSC & 35 & $2 \%$ & Winter Simulation Conference \\
\hline DIS & 32 & $2 \%$ & ACM conference on Designing Interactive Systems \\
\hline Mobile HCI & 32 & $2 \%$ & $\begin{array}{l}\text { International Conference on Human-Computer Interaction with Mobile Devices and } \\
\text { Services }\end{array}$ \\
\hline
\end{tabular}

Tabela 2. Referências mais usadas entre artigos sobre affordances. Fonte: autor

\begin{tabular}{llr}
\hline ID & Referência & n. cit. \\
\hline A1 & Ishii and Ullmer. 1997. "Tangible bits: towards seamless interfaces between people, bits and atoms & 42 \\
\hline A2 & Norman. 1999. "Affordance, conventions, and design”. & 38 \\
\hline A3 & Gaver 1991. "Technology affordances". & 34 \\
\hline A4 & Dourish, 2004. "Where the action is: the foundations of embodied interaction”. & 24 \\
\hline A5 & Scott, Sheelagh and Inkpen, 2004. "Territoriality in collaborative tabletop workspaces." & 16 \\
\hline A6 & Papert, "Mindstorms: children, computers, and powerful ideas”. & 16 \\
\hline A7 & Salen, Zimmerman, 2003. "Rules of Play: Game Design Fundamentals" & 16 \\
\hline A8 & Gibson (1979). "The ecological approach to visual perception". & 14 \\
\hline A9 & Suchman, "Plans and situated actions: the problem of human-machine communication” & 14 \\
\hline A10 & Fitzmaurice, Ishii, and Buxton. 1995. "Bricks: laying the foundations for graspable user interfaces” & 14 \\
\hline
\end{tabular}

O conceito de affordance foi proposto inicialmente por Gibson (A8) como parte de um estudo psicológico sobre a percepção do ambiente por pessoas e animais usando uma abordagem ecológica. Para ele, affordances são tudo aquilo que o ambiente oferece ao animal como possibilidade de ação, estabelecendo relações que dependem das características físicas do ambiente e das capacidades do animal. Sua existência independe de serem percebidas de forma consciente, ou das intenções do sujeito. A partir da proposta de Gibson, Gaver (A3) apresenta uma visão mais voltada para a tecnologia, discutindo como ações complexas podem ser desempenhadas de forma sequencial ou aninhada de interações mais simples. Gaver se apoia em um trabalho anterior de Norman (Norman, 1988) voltado ao design de objetos, mas sem muita ênfase. Sua principal contribuição foi mostrar, à época, que a ideia de affordances poderia ser aplicada ao design de interfaces de sistemas computacionais para torná-las mais fáceis de aprender e usar. Por exemplo, o desenho de um botão com sensação de relevo na tela indicaria às pessoas que ali pode ser clicado.

O texto localizado de Norman (A2) propôs uma aplicação do conceito para o design de objetos em geral, e posteriormente para a interface sistemas computacionais. No artigo citado, ele traz um histórico de como o termo foi aplicado inicialmente para entender como pessoas podem interagir com objetos e aprender a operar dispositivos pela primeira vez, sem conhecimento anterior: a aparência do objeto proporcionaria dicas sobre as possibilidades de interação (Norman, 1988). Posteriormente, chamou de "affordances percebidas" as possibilidades de interação que o usuário de um objeto percebe como possíveis, independentemente de elas existirem de fato, podendo também existirem sem ser percebidas. Por exemplo: para Norman, o usuário pode clicar em qualquer lugar de uma tela de computador, mas somente em regiões que proveem algum tipo de 
feedback o usuário perceberá que esta ação é significativa - como em widgets de botões, que além de relevo, têm o efeito de "abaixar" ou "serem acionados" quando clicados. Mais recentemente, Dourish (A4) volta a direcionar o interesse para objetos em geral e sua interação com pessoas, sem um foco específico em interfaces de usuário, mas sim nas possiblidades que sistemas interativos acrescentam às nossas atividades cotidianas. Não é apresentada nenhuma nova definição para affordance, apoiando sua discussão nas propostas anteriores de Gibson, Gaver e Norman. Contudo, reforça o conceito de que uma affordance é uma relação ternária entre um organismo, o seu ambiente, e a possibilidade de uma ação em particular.

Os artigos de Ishii et al. (A1) e de Fitzmaurice et al. (A10) evidenciam a aplicação de affordances na compreensão da interação com objetos físicos e sua utilidade para o desenvolvimento de interfaces tangíveis. Na visão de Ishii e seus coautores, a adoção de objetos físicos para interação enriquece as affordances dos sistemas construídos, ao permitir ao usuário utilizar uma maior variedade de sentidos, sensações e habilidades físico-motoras do que seria possível em um display gráfico.

\subsection{Normas Sociais}

Foram encontrados 1504 artigos $\left(\mathrm{I}_{\mathrm{N}}\right)$, cuja distribuição por ano de publicação é mostrada na Figura 2 (direita). As dez conferências ou periódicos em que foram encontrados com mais frequência são listados na Tabela 3. A partir deste conjunto, foram localizadas 10481 referências distintas $\left(\mathrm{D}_{\mathrm{N}}\right), 648$ delas sendo citadas por mais de um artigo do conjunto inicial. Muitos destes artigos e referências usam "norma" no sentido matemático do termo - função de distância ou comprimento - e foram descartados na composição de $\mathrm{S}_{\mathrm{N}}$. Os dez artigos mais citados (incluindo um total de 16 artigos devido ao empate no número de citações) são mostrados na Tabela 4 a seguir. Podemos agrupá-los por tema: teoria de normas (N8, N11, N13, N4, N10), sistemas multi-agentes (N4, N8 e N10), privacidade online (N1, N7), design (N12, N14, N15). As referências N2, N3, N5, N6, N9 e N16 não abordam formalmente o conceito de norma e não serão descritas.

Tabela 3. As 10 conferências ou periódicos mais encontrados na busca por normas. Fonte: autor

\begin{tabular}{llll}
\hline Sigla & Qtd. & $\%$ & Nome do periódico/conferência \\
\hline AAMAS & 169 & $11 \%$ & International Conference on Autonomous Agents and Multiagent Systems \\
\hline Mach. Learn. Res. & 117 & $8 \%$ & Journal of Machine Learning Research \\
\hline SODA & 64 & $4 \%$ & ACM-SIAM Symposium on Discrete Algorithms \\
\hline CHI & 62 & $4 \%$ & ACM CHI Conference on Human Factors in Computing Systems \\
\hline CSCW & 62 & $4 \%$ & ACM Conf. on Computer-Supported Cooperative Work and Social Computing \\
\hline STOC & 61 & $4 \%$ & ACM Symposium on Theory of Computing \\
\hline ICAIL & 57 & $4 \%$ & International Conference on Artificial Intelligence and Law \\
\hline CHI EA & 41 & $3 \%$ & CHI Extended Abstracts \\
\hline KDD & 39 & $3 \%$ & Conference on Knowledge Discovery and Data Mining \\
\hline WI-IAT & 32 & $2 \%$ & Int. Conf. on Web Intelligence, and the Int. Conf. on Intelligent Agent Technology \\
\hline
\end{tabular}

Tabela 4. Referências mais usadas entre artigos sobre normas. Fonte: autor

\begin{tabular}{llr}
\hline ID & Referência & $\begin{array}{r}\text { n. } \\
\text { cit. }\end{array}$ \\
\hline N1 & Nissenbaum, 2009. "Privacy in context: Technology, policy, and the integrity of social life”. & 7 \\
\hline N2 & $\begin{array}{l}\text { Davis, 1989. "Perceived usefulness, perceived ease of use, and user acceptance of information } \\
\text { technology." }\end{array}$ & 6 \\
\hline N3 & Winograd and Flores, 1987. "Understanding Computers and Cognition: A New Foundation for Design” & 6 \\
\hline N4 & Vasconcelos, Kollingbaum and Norman, 2009. "Normative conflict resolution in multi-agent systems." & 5 \\
\hline N5 & Braun and Clarke, 2006. "Using thematic analysis in psychology." & 5 \\
\hline N6 & Davis and Warshaw, 1989. "User acceptance of computer technology: a comparison of two theoretical & 5 \\
\hline N7 & Palen and Dourish, 2003. "Unpacking privacy for a networked world." & 5 \\
\hline N8 & Sen and Airiau, 2007. "Emergence of norms through social learning." & 4 \\
\hline N9 & Butler, 1990. "Gender trouble: feminism and the subversion of identity". & 4 \\
\hline
\end{tabular}




\begin{tabular}{lll}
\hline N10 & Artikis, Pitt, and Sergot, 2002. "Animated specifications of computational societies." & 4 \\
\hline N11 & Jones and Sergot, 1996. "A formal characterisation of institutionalised power." & 4 \\
\hline N12 & Sengers, Boehner, David and Kaye. 2005. "Reflective design.". & 4 \\
\hline N13 & $\begin{array}{l}\text { Shoham and Tennenholtz, 1997. "On the emergence of social conventions: modeling, analysis, and } \\
\text { simulations." }\end{array}$ & 4 \\
\hline N14 & Fogg, 2002. "Persuasive technology: using computers to change what we think and do." & 4 \\
\hline N15 & $\begin{array}{l}\text { Consolvo, Everitt, Smith and Landay, 2006. "Design requirements for technologies that encourage } \\
\text { physical activity". }\end{array}$ & $\begin{array}{l}\text { Marwick and Boyd, 2011. "I tweet honestly, I tweet passionately: Twitter users, context collapse, and the } \\
\text { imagined audience." }\end{array}$ \\
\hline N16
\end{tabular}

Os textos de Sendip de Airiau (N8) e o de Shoham e Tenneholtz (N13) propõem hipóteses para os mecanismos de surgimento de normas em uma sociedade, ambos com apoio de teoria dos jogos e simulações computacionais. A definição de norma dada por Sendip em N8 é a de uma opção de comportamento dos indivíduos de uma sociedade visando aumentar os benefícios obtidos durante interações entre eles. Segundo os autores, normas evoluem de baixo para cima (bottom-up) a partir de expectativas de comportamento criadas na interação com outros indivíduos. O texto N8 baseia-se no referencial teórico de Lewis (Lewis, 1969) e utiliza uma simulação com agentes computacionais, onde nenhum agente tem conhecimento sobre os estados internos dos demais, e todo conhecimento e decisões dependem de informação obtida durante as interações. Também Shoham e Tenneholtz (N13) visam entender a emergência de normas pela maximização de recompensas acumuladas ao longo de interações. Definem uma lei social, que é uma restrição imposta por uma autoridade central nas possibilidades de ação disponíveis aos agentes.

$\mathrm{O}$ artigo de Artkis et al. (N10) analisa aspectos institucionais e sociais de sistemas computacionais abertos, como e-markets e protocolos de gerenciamento de direitos autorais de mídia. Normas são tratadas como obrigações, proibições e permissões promovidas por meio de sanções. Traz a definição de Searle (1965) de fatos brutos e institucionais; por exemplo, uma pessoa estar segurando fisicamente um objeto é um fato bruto, enquanto que o reconhecimento da posse do objeto é um fato institucional, circunscrito à instituição ou sociedade em que é analisado. Por fim, apresentam uma ferramenta para modelar normas e simular a interação de software com sujeitos que podem ou não segui-las; permitindo aos projetistas decidem se é desejável ou não implantar tais sistemas, a fim de antecipar possíveis novos comportamentos indesejados, seja do tipo mais individualistas ou exageradamente competitivos.

Jones e Sergot (N11) trazem uma discussão que liga as normas de uma instituição ao poder de seus agentes, definindo conceitos como poder institucionalizado, poder legal, permissão, possibilidade prática, autorização e delegação: ter permissão para fazer algo é diferente de ter o poder de fazê-lo, se a instituição não proporcionar na prática a possiblidade de desempenhar as ações requeridas. Vasconcelos et al. (N4) também definem norma como permissões, proibições e obrigações, de modo que agentes vão perseguir suas obrigações, evitando proibições e tirando proveito de suas permissões. Neste artigo, os autores apresentam uma representação formal de normas, contendo a identificação de uma ação, um agente, um papel e um instante no tempo. Discutem como normas interagem podendo gerar conflitos, onde um mesmo agente pode ser ao mesmo tempo obrigado e proibido de desempenhar uma ação, porém sujeitos que violam uma norma estão sujeitos a sanções; são apresentadas estratégias para sua solução. Pressão e suporte sociais são também tratados pelo artigo de Consolvo et al. (N15).

O livro de Nissenbaum (N1) estuda privacidade em meios digitais, propondo um baseado no contexto social governado por normas específicas e que governa as atividades das pessoas, o fluxo de informações, e os papéis desempenhados. Ainda tratando de privacidade, Palen e Dourish mostram em N7 como um processo dinâmico e dialético, com a fronteira entre público e privado sendo constantemente renegociado de acordo com as circunstâncias. Recorda que nossas formas mais familiares de gerenciar a privacidade dependem fundamentalmente de características físicas do ambiente - como nossa incapacidade de enxergar através de portas fechadas - e normas comportamentais sobre manutenção do espaço interpessoal e respeito por propriedade alheia, por exemplo. Em contextos digitais, entretanto, muitas destas restrições não estão disponíveis. Sengers et al. (N12) lembram que decisões de design, tomadas consciente ou inconscientemente, moldam as experiências diárias das pessoas. Chama atenção para a responsabilidade do designer em relação ao impacto de seus produtos, ao mesmo tempo em que lembra que o usuário não é um receptor passivo de tecnologia, mas sim um sujeito ativo que é capaz de fugir às normas de comportamento socialmente estabelecidas e propor novas finalidades para os artefatos construídos. 
Fogg (N14), em seu livro sobre design persuasivo, traz um capítulo dedicado a "computadores como atores sociais". O autor afirma que não apenas pessoas reagem a computadores como se estes fossem seres vivos, mas como se fossem parte de um mesmo grupo social. Estas reações podem ser obtidas por meios físicos, psicológicos, pela linguagem, pela dinâmica social ou por papéis definidos em um grupo. Um computador enquanto ator social pode moldar o comportamento de pessoas, por exemplo, provendo recompensas para usuários que se comportam de uma maneira desejada, demonstrando "traços de personalidade", ou assumindo um papel de autoridade.

\subsection{Trabalhos Relacionados a Ambos os Conceitos}

Treze publicações pertencem à intersecção entre ambas as buscas $\left(I_{I}=I_{A} \cap I_{N}\right)$. Duas foram descartadas pois as palavras da busca apenas aparecem no abstract, sem utilizar de fato os conceitos. Um terceiro foi descartado por ser um short paper com pouco conteúdo, o que inviabilizou sua análise; restou assim um conjunto $\mathrm{D}_{\mathrm{I}}$ com 10 textos. Diferentemente do procedimento adotado nas seções anteriores, aqui analisaremos os próprios artigos encontrados $\left(\mathrm{D}_{\mathrm{I}}\right)$, e não suas referências $\left(\mathrm{S}_{\mathrm{I}}\right)$, já que poucas referências se repetem - em particular, A3. Este conjunto é mostrado integralmente na Tabela 5 e foi agrupado por temas: redes sociais (I5, I6, I7, I10), jogos (I3, I8, I9), construção de conhecimento online (I1, I4) e webservices (I2).

Tabela 5. Trabalhos pertencentes à intersecção das buscas. Fonte: autor

\begin{tabular}{lllr}
\hline ID & Publicação & Conferência & Ano \\
\hline I1 & $\begin{array}{l}\text { X. Tang, J. Coffey: Fostering Meaningful Scientific Argumentation } \\
\text { Practices Through Ongoing Classroom Interactions }\end{array}$ & $\begin{array}{l}\text { Int. Conf. on Learning } \\
\text { Sciences }\end{array}$ & 2010 \\
\hline I2 & $\begin{array}{l}\text { A. Benfell: A Multi-responsive Communication Architecture for Web } \\
\text { Service Description and Discovery }\end{array}$ & I-SEMANTICS & 2010 \\
\hline I3 & $\begin{array}{l}\text { D. Wilde: hipDisk: Experiencing the Value of Ungainly, Embodied, } \\
\text { Performative, Fun }\end{array}$ & CHI Extended Abstracts & 2012 \\
\hline I4 & $\begin{array}{l}\text { T. Kriplean, J. Morgan, D. Freelon, A. Borning and L. Bennett: } \\
\text { Supporting Reflective Public Thought with Considerate }\end{array}$ & ACM CSCW & 2012 \\
\hline I5 & $\begin{array}{l}\text { R. Bodle: The Ethics of Online Anonymity or Zuckerberg vs. Moot } \\
\text { I6 }\end{array}$ & $\begin{array}{l}\text { B. Xu, P. Chang, C. Welker, N. Bazarova, D. Cosley: Automatic } \\
\text { Archiving Versus Default Deletion: What Snapchat Tells Us About }\end{array}$ & ACM CSCW \\
\hline $\begin{array}{l}\text { Ephemerality in Design; } \\
\text { I7 }\end{array}$ & $\begin{array}{l}\text { J. Groshek and E. Tandoc: The Affordance Effect: Gatekeeping and } \\
\text { (Non)Reciprocal Journalism on Twitter }\end{array}$ & Social Media \& Society & 2013 \\
\hline I8 & $\begin{array}{l}\text { P. Alavesa, M. Pakanen, H. Kukka, M. Pouke and T. Ojala: Anarchy } \\
\text { or Order on the Streets: Review Based Characterization of Location } \\
\text { Based Mobile Games }\end{array}$ & CHI PLAY & 2016 \\
\hline L9 & $\begin{array}{l}\text { L. Poretski, J. Lanir and O. Arazy: Normative Tensions in Shared } \\
\text { Augmented Reality }\end{array}$ & ACM CHI & 2017 \\
\hline I 10 & $\begin{array}{l}\text { M. Devito, A. Walker and J. Birnholtz: Too Gay for Facebook': } \\
\text { Presenting LGBTQ+ Identity Throughout the Personal Social Media } \\
\text { Ecosystem }\end{array}$ & ACM CHI & 2018 \\
\hline
\end{tabular}

Bodle (I5), tomando como base o Facebook, afirma que o anonimato online está se tornando cada vez mais difícil de se manter. Affordances são usadas no sentido de aquilo que o anonimato online é capaz de proporcionar, ou seja, reduzir a responsabilidade, aumentar a desinibição, e tem efeito de despersonalização. Embora estudos anteriores sugeriam que, em contextos anônimos, as pessoas eram mais propensas a se comportar de forma anti-normativa, em I5 é apresentado que em comunidades online uma redução na individuação pode contribuir para um forte senso de identidade coletiva. No artigo I6 é discutido o alinhamento entre o que foi chamado de uma affordance do Snapchat, que é sua funcionalidade padrão de apagar fotos alguns segundos após serem visualizadas, com a norma social de não se fazer cópias de tela (printscreen) do aplicativo. Esta norma é parcialmente respeitada, e isto é discutido com base na teoria de Nissenbaum (Nissenbaum, 2009) que postula que normas são altamente dependentes de contexto e que os indivíduos entram e saem de contextos distintos que apresentam diferentes normas, mesmo durante o uso de um único aplicativo. 
Em I7, o termo "technological affordance" é usado com base no referencial de Halpern e Gibbs (2013), que por sua vez se baseia em Gibson (texto A8), porém de forma genérica, considerando qualquer capacidade de interação ou atuação fornecida pelos sistemas, como o anonimato. Devito et al. (I10) argumentam que é importante considerar as decisões do usuário sobre sua auto-apresentação como parte de um ecossistema, em que as plataformas de mídia social, suas audiências, affordances técnicas e normas comportamentais são componentes. No grupo de jogos, o artigo I3 mostra um dispositivo cujas affordances físicas levam o jogador a fazer movimentos "socialmente estranhos", ou seja, leva o jogador a desafiar o comportamento estabelecido normativamente. Em I8 é mais uma vez citado o referencial de Norman (1988), usado para se referir à "liberdade de movimento" proporcionada por um jogo para dispositivos móveis. No caso, estes jogos devem garantir que seus usuários continuam seguindo normas sociais, respeitando leis, e ficar seguro. $\mathrm{O}$ artigo $\mathrm{I} 9$ afirma que uma realidade aumentada compartilhada possui affordances que podem transformar algo que pareceria rude em ambientes sociais tradicionais em algo visto como normal e aceitável.

Baseado na Teoria da Atividade (Engestrom, 1987), o artigo I1 estuda as normas sociais de interação em uma sala de aula, em momentos em que deve haver argumentação e formação de sentido. Afirma que devem haver mediadores com affordances específicas para favorecer este comportamento. $\mathrm{O}$ artigo de Kriplean $e t$ al. (I4) mostra como normas sociais vigentes influenciam em discussões políticas online. Com base nos princípios da Web Pragmática, o artigo I2 propõe um mecanismo que captura padrões comportamentais de serviços usando simultaneamente affordances e normas que, quando mesclados, formam uma arquitetura de comunicação para a descoberta de webservices; utilizam o referencial de Liu e Li (2014) que articula os conceitos de affordances e normas em sistemas de informação.

\section{AFFORDANCES E NORMAS À LUZ DE PESQUISAS RECENTES}

Embora affordances tenham sido postuladas por Gibson de forma qualitativa, resultados recentes da neurociência corroboram de forma quantitativa sua existência e revelam seu mecanismo de funcionamento. Murata et al. (2016) utilizaram medidas da frequência de acionamento de neurônios no córtex de primatas para mostrar que existem áreas do córtex - conhecidas como circuito AIP-F5 - responsáveis por correlacionar características físicas específicas de objetos com os movimentos necessários para manipulá-los. Neurônios diferentes são acionados para diferentes formas, orientações e tamanhos de objetos. Porém, os mesmos neurônios são ativados quando o objeto é apenas visualizado e quando ele é manipulado, mostrando que nesta área ocorre a correlação entre a percepção visual do objeto e as possibilidades de interação com o mesmo. Isto foi denominado "vocabulário motor". Ou seja, existe uma base neuronal para a relação entre a percepção visual de características físicas de um objeto e a ativação de áreas que preparam o sujeito para ação adequada na interação com tal objeto - o que é bastante alinhado com a proposição original de affordance. Outro ponto importante é a descoberta de que, pelo ponto de vista de impulsos entre neurônios, quando usamos uma vareta para explorar objetos presentes em um espaço físico delimitado, a vareta passa a ser compreendida como se fosse parte do braço (Murata et al., 2016). Isso demostra que o cérebro se adequa à realidade do corpo, no caso, considerando sua "extensão" com uma ferramenta. Progressivamente, este novo sujeito híbrido composto por mão + vareta estabelece suas próprias affordances com o ambiente com o qual interage, o que seria um indicativo que as mesmas não seriam inatas ou fixas, mas podem ser aprendidas ou desenvolvidas.

Já em relação a normas, Centola et al. (2018) argumentam que quando uma minoria comprometida atinge um tamanho crítico de grupo, o sistema social cruza um ponto crítico; uma vez atingido o ponto de inflexão - no original, tipping point - as ações de um grupo minoritário desencadeiam uma cascata de mudança de comportamento que aumenta rapidamente a aceitação de uma nova forma de agir. De acordo com esta visão, o poder de pequenos grupos não vem de sua autoridade ou riqueza, mas de seu compromisso com a causa. Muitos estudos a este respeito se basearam e simulações computacionais empregando teoria dos jogos para demonstrar esta dinâmica. O que Centola et al. (2018) trazem de novo é ter registrado e mensurado este comportamento em 10 comunidades reais, com 20 a 30 pessoas cada uma. Uma norma dominante era deliberadamente desafiada por sujeitos participantes do experimento. Em comunidades em que o número de desafiantes era inferior a $25 \%$ do total, apenas um pequeno número de outros membros era convertido para o comportamento alternativo e, mesmo assim, ao longo do tempo, acabavam por retornar ao comportamento dominante. Já para uma minoria entre $25 \%$ e $30 \%$ do grupo, em todos os grupos observados 
o comportamento alternativo se tornou norma dentro da janela de tempo do estudo. Ou seja, este estudo valida as diversas simulações computacionais feitas sobre o estabelecimento de novas normas em um grupo social, além de definir um ponto de inflexão em $30 \%$ da população, a partir do qual as interações levam os praticantes da norma antiga se converterem à nova norma.

\section{DISCUSSÃO}

Fica claro que há evidências empíricas quanto à existência de estruturas mentais específicas para a relação entre corpo e objetos, mediada pela percepção que se tem de ambos. Do ponto de vista de paradigma científico, Gibson procurou superar a dicotomia objetivista-subjetivista (Kaptelinin e Nardi, 2012) vigente em áreas como psicologia e filosofia, e foi adotado para direcionar o design de artefatos físicos ou computacionais. Desta forma, na proposta original de Gibson (A8), uma affodance pode ser vista apenas como uma hipótese, não algo que se possa determinar a existência. Norman (A2) inclui a percepção em sua definição, tornando mais específicos o seu significado e possibilidades.

Osch e Mendelson (2011) lembram que existe uma segunda perspectiva necessária a compreensão de affordances, principalmente para Interação Humano-Computador: a do designer. Ele ou ela pode ter a intenção de aplicar determinadas características a um artefato sendo planejado ou construído, de modo a proporcionar uma possiblidade de interação; porém pode não ser bem-sucedido. Inversamente, uma affordance pode ser percebida pelo usuário sem ter sido intenção do designer - como em usos não planejados de artefatos. Além disso, pode ser intenção do designer que certas affordances não sejam perceptíveis - como easter eggs em jogos, por exemplo. Por fim, podem existir affordances emergentes, ou seja, que não foram intencionadas pelo designer nem percebidas diretamente pelo usuário, mas que surgem durante a interação; neste caso, é necessário um avaliador externo para perceber que a presença do artefato provocou uma alteração nas ações do usuário.

O processo de emergência de comportamentos decorrentes da interação entre o artefato e o usuário pode ser compreendido como uma contribuição do artefato em si. Este estudo pode ser feito por pontos de vista como o de Suchmann (A9), ou outras fontes de teorias sobre a sociomaterialidade, como Orlikowski (2007) ou Latour (2005), entre outros. Em comum, estas abordagens admitem o papel ativo de artefatos moldando e direcionando ações humanas, inclusive interações sociais. Embora sem um aprofundamento teórico formal, este papel já é explorado por Fogg (N14). Normas sociais emergem quando o comportamento de um indivíduo é percebido como repetitivo em outros do mesmo grupo. Artefatos computacionais podem interferir nesta percepção e, desta forma, atuar de forma favorável ou contrária a determinados comportamentos - seguindo ou não uma intenção de design. Porém, para o conceito de normas, além da falta de uma referência canônica para a sua definição, notou-se duas abordagens principais: a do grupo que procura entender como surgem normas e ordem em grupos de agentes independentes, e a do grupo que já assume a existência de pressões sociais - representáveis por meio de lógica deôntica, por exemplo. Há uma lacuna na ligação entre estes dois movimentos, mostrando como normas emergentes se tornam consolidadas. A demonstração experimental do tipping point, no mundo real, preenche parcialmente esta lacuna.

No estudo da intersecção também é possível verificar que são todos artigos recentes, publicados a partir de 2010, o que demonstra sua relevância atual, principalmente se considerarmos problemas como questões de gênero, ética em redes sociais, e o futuro da inteligência artificial (Yu et al., 2018; Chung et al., 2017). A análise das conferências e periódicos onde os textos originais foram encontrados (Tabelas 1 e 3 ) mostra sua importância para várias áreas de pesquisa como interfaces e interação (IHC e TEI), trabalho colaborativo (CSCW), agentes autônomos e inteligentes (AAMAS e WI-IAT); o que pode ser expandido para novas áreas como Ambient Assisted Living - AAL.

Resumindo, o que ambos os conceitos tratam é a influência que objetos ou pessoas exercem na disposição para ação de um sujeito. Propomos compreendê-los em um contínuo, que vai desde o que é naturalmente determinado até o que é socialmente definido. Interagem neste percurso a construção do conhecimento e a produção de tecnologia, em um ciclo que se realimenta. Este tema constitui o foco dos desdobramentos do presente trabalho, em particular, a compreensão de objetos e pessoas conectados e computacionalmente aparelhados, e suas mútuas interferências. 


\section{CONSIDERAÇÕES FINAIS}

Neste artigo, analisamos as fontes bibliográficas mais referenciadas por trabalhos que abordam os conceitos de affordances e normas. De início, esperamos ter contribuído com uma fonte concisa e simplificada, em língua portuguesa, de referenciais teóricos relevantes para dois dos conceitos mais importantes para pesquisa em IHC e design de aplicações e dispositivos com viés social. Em particular, a intersecção entre affordances e normas, representada pelos artigos elencados na seção 3.3, mostra a atualidade do tema, tendo em vista tratar-se de artigos recentes. Entretanto, também mostra que os conceitos têm sido usados de forma menos rigorosa, o que pode indicar uma lacuna para novos referenciais.

Para o futuro, podemos cogitar que a união dos dois conceitos poderá proporcionar uma simplificação paradigmática útil para Interação Humano-Computador em desafios relacionados à ubiquidade, múltiplos dispositivos e tangibilidade, que requerem um referencial teórico que contemple a sociomaterialidade em sua essência. A neurociência pode fornecer novas formas de compreender a ligação entre objetivo e subjetivo, bem como a relação entre indivíduo e sociedade. E a sociologia, em particular as vertentes que reconhecem o papel de objetos físicos nas relações sociais (Osch e Mendelson, 2011), ajudar na compreensão do papel de artefatos digitais em padrões de comportamento.

\section{AGRADECIMENTO}

O autor agradece o apoio financeiro recebido da EDUCORP/Unicamp.

\section{REFERÊNCIAS}

Birnbaum, Gurit E. , Moran Mizrahi, Guy Hoffman, Harry T. Reis, Eli J. Finkel, and Omri Sass, 2016. Machines as a Source of Consolation: Robot Responsiveness Increases Human Approach Behavior and Desire for Companionship. In The Eleventh ACM/IEEE International Conference on Human Robot Interaction (HRI '16). IEEE Press, Piscataway, NJ, USA, 165-171.

Brooks, Rodney, 1991. New approaches to robotics. Science 253, no. 5025: 1227-1232.

Centola, Damon, Joshua Becker, Devon Brackbill, and Andrea Baronchelli, 2018. Experimental evidence for tipping points in social convention. Science 360, no. 6393: 1116-1119.

Chung, Hyunji , Michaela Iorga, Jeffrey Voas, and Sangjin Lee, 2017. Alexa, can I trust you? Computer 50, no. 9: 100-104.

Dourish, P., 2004. What we talk about when we talk about context. Personal and ubiquitous computing 8, no. 1: 19-30.

Engestrom, Y., 1987. Learning by expanding. Orienta-konsultit, Helsinki.

Halpern, Daniel and Gibbs, Jennifer, 2013. Social media as a catalyst for online deliberation? Exploring the affordances of Facebook and YouTube for political expression. Computers in Human Behavior, 29, 1159-1168

Kaptelinin, Victor and Nardi, Bonnie, 2012. Affordances in HCI: toward a mediated action perspective. In Proceedings of the SIGCHI Conference on Human Factors in Computing Systems, pp. 967-976. ACM, 2012.

Latour, Bruno, 2005. Reassembling the social: An introduction to actor-network theory. Oxford: Oxford University Press, pp. 801-831.

Lewis, David, 1969. Convention: A Philosophical Study, Harward University Press.

Liu, Kecheng and Li, Weizi, 2014. Organisational Semiotics for Business Informatics. Taylor and Francis, Hoboken.

Murata, Akira, Wen Wen, and Asama, Hajime, 2016. The body and objects represented in the ventral stream of the parieto-premotor network. Neuroscience research 104: 4-15.

Nissenbaum, Helen, 2009. Privacy in context: Technology, policy, and the integrity of social life. Stanford University Press.

Norman, Donald A., 1988. The psychology of everyday things. New York: Basic books.

Orlikowski, Wanda, 2007. Sociomaterial practices: Exploring technology at work. Organization Studies (28:9), pp. 1433-1448.

Osch, Wietske and Mendelson, Orr, 2011. A typology of affordances: Untangling sociomaterial interactions through video analysis. Proceedings of the International Conference on Information Systems, ICIS 2011, Shanghai, China, December 4-7, 2011.

Searle, John R., 1965. What is a speech act. Perspectives in the philosophy of language: a concise anthology 2000: 253-268.

Yu, Han, Zhiqi Shen, Chunyan Miao, Cyril Leung, Victor R. Lesser, and Qiang Yang, 2018. Building ethics into artificial intelligence. Proceedings of the 27th International Joint Conference on Artificial Intelligence. Stockholm, Sweden July $13-19,2018$ 